\title{
Aggregating Fuzzy QL- and (S,N)-Subimplications: Conjugate and Dual Constructions ${ }^{\dagger}$
}

\author{
R.H.S. REISER ${ }^{1 *}$, I.C.K. BENÍTEZ ${ }^{1}$, A.C. YAMIN ${ }^{1}$ and B.R.C. BEDREGAL ${ }^{2}$
}

Received on September 12, 2014 / Accepted on September 1, 2015

\begin{abstract}
S,N)- and QL-subimplications can be obtained by a distributive $n$-ary aggregation performed over the families $\mathcal{T}$ of t-subnorms and $S$ of t-subconorms along with a fuzzy negation. Since these classes of subimplications are explicitly represented by $\mathrm{t}$-subconorms and $\mathrm{t}$-subnorms verifying the generalized associativity, the corresponding (S,N)- and QL-subimplicators, referred as $I_{S, N}$ and $I_{S, T, N}$, are characterized as distributive $n$-ary aggregation together with related generalizations as the exchange and neutrality principles. Moreover, the classes of $(\mathrm{S}, \mathrm{N})$ - and QL-subimplicators are obtained by the median operation performed over the standard negation $N_{S}$ together with the families of t-subnorms and t-subconorms by considering the product t-norm $T_{P}$ as well as the algebraic sum $S_{P}$, respectively. As the main results, the family of subimplications $I_{S_{P}, N}$ and $I_{S_{P}, T_{P}, N}$ extends the corresponding classes of implicators by preserving their properties, discussing dual and conjugate constructions.
\end{abstract}

Keywords: median aggregation, t-sub(co)norms, fuzzy (sub)implications, QL-implications, (S,N)-implications.

\section{INTRODUCTION}

The study of aggregation operators is a large domain, supported by using aggregation concepts modeling uncertainty in distinct fields such as social, engineering or economical problems which are based on fuzzy logic (FL) [1, 2, 3, 4]. Consequently, they have been applied to many fields of approximate reasoning [5], e.g. image processing, data mining, pattern recognition [6, 7], fuzzy relational equations and fuzzy morphology $[8,9,10]$.

Despite the applications of aggregation operators in many potential distinct areas, this paper deals with the current status of the theory of aggregation operators in FL and also considers some

\footnotetext{
*Corresponding author: Renata Reiser.

$\dagger$ This work is supported by the Brazilian funding agencies CAPES, FAPERGS (Ed. PqG 06/2011, under the process number 11/1520-1). This article extends the work published by IEEE Xplore Pos-Proceedings of WEIT 2013, which was indicated as a select paper to TEMA.

${ }^{1}$ Centro de Desenvolvimento Tecnológico, Universidade Federal de Pelotas, 96010-610 Pelotas, RS, Brasil. E-mails: reiser@inf.ufpel.edu.br; ickbenitez@Informationufpel.edu.br; yamin@Informationufpel.edu.br

2 Departamento de Informática e Matemática Aplicada, Universidade Federal do Rio Grande do Norte, 59078-970 Natal, RN, Brasil. E-mail: bedregal@ dimap.ufrn.br
} 
of their main properties: symmetry, monotonicity, idempotency, homogeneity and distribution. Moreover, many other extensions of fuzzy logic make use of aggregation operators as pointed out in, e.g. Interval-valued Fuzzy Logic [11, 12, 13, 14, 15], Intuitionistic Fuzzy Logic [11, 16, 17, 18] and Hesitant Fuzzy Logic [19, 20].

Distinguished classes of aggregations have been studied in the literature, e.g. the average, the conjunctive and the disjunctive, as well as some classical generalizations like the (ordered) weighted mean and the k-order statistics. This work considers the median average aggregation [21], which is applied into a family of fuzzy connectives to generate new fuzzy connectives, preserving the same properties verified by the family.

As a novel theoretical result, our procedure to obtain new fuzzy connectives is not restricted to binary aggregations, performing a two-by-two aggregation process on multiple input values. By applying the median (as general $n$-ary aggregation) to families of fuzzy connectives, we are able to generate new members of such families preserving their main properties. So, performing the median by means of associative and commutative fuzzy connectives, the interchange of the multiple input values is allowed. Moreover, by invoking the so-called self-dual fuzzy connective, we ensure aggregations of complementary values as complements of the original ones, establishing new results from $n$-ary connectives as self-dual operators. In preference modeling and multicriteria decision-making, self-dual n-ary aggregation operators ensure that individual, reciprocal preference relations are combined collectively, preserving reciprocal preference relations.

Following the studies presented in [22, 23, 24], by relaxing the neutral element property related to triangular (co)norms, the class $\mathcal{T}(S)$ of $t$-sub(co)norms is considered. Recently, an increasing number of papers regarding various aspects of t-subnorms has appeared, as evidence of their importance in many other related research topics. See, e.g. the problem of construction of leftcontinuous t-norms [26]. In a more general algebraic context, the relationship between special classes of t-subnorms and ordinal sums of semigroups was recently clarified [5]. Continuous triangular subnorms are shown to be the ordinal sum of Archimedean continuous t-subnorms with at most one proper t-subnorm [23] enabling extensions of t-subnorms on bounded lattices via retraction operators.

As a consequence of this slight modification of such neutral element axiom of triangular (co)norms, it leads to a more general definition of sub(co)implications by relaxing the boundary condition $I(1,0)=0$. Thus, the fuzzy $(\mathrm{S}, \mathrm{N})$-subimplication class, explicitly represented by negations and fuzzy t-subconorms is considered in this paper, including their dual constructions. In particular, generalizations of well-known operators product t-norm and probabilistic sum are taken into account and provide interesting examples based on the median aggregation operator. Since this study considers $n$-ary aggregations, generalized associativity, exchange principle and distributivity properties also need to be considered.

In [24], the class $\mathcal{J}$ of fuzzy QL-subimplications is introduced, which is obtained by the median aggregation performed over a family of t-sub(co)norms $\mathcal{T}(S)$ along with fuzzy negations. These results state the following constructions as equivalent: 
(i) Firstly, we can aggregate all the $t$-sub(co)norms $\left(T_{i}\left(S_{i}\right)\right)$ of family $\mathcal{T}(S)$ and then generate a class $\mathcal{I}_{Q L}$ of QL-subimplications;

(ii) Secondly, in other order, we can obtain each QL-subimplication which is expressed by composition of a $t$-sub(co)norm and a fuzzy negation and then, by aggregating all the QL-subimplications related to the median we obtain the same class $\mathcal{I}_{Q L}$.

Analogously, by results in [22], (S,N)- and R-implications are generated by the aggregation of t-sub(co)norms and fuzzy negations.

As the main contribution, in this work, the converse construction presented in [24] is now considered by stating the conditions under which (S,N)- or QL-subimplications can be extended in order to obtain the corresponding $(\mathrm{S}, \mathrm{N})$ - or QL-implications. We also discuss the $N$-dual and conjugate constructions of aggregate operators. Extensions of fuzzy connectives, dual constructions and conjugate functions are relevant operators in order to generate new fuzzy connectives, preserving their main properties on the unit interval. In this paper, the authors make use of commutative diagrams to illustrate that the composition between the dual operator and conjugate $(\mathrm{S}, \mathrm{N})$ - or QL-subimplications obtained by the median aggregation operator is preserved in such classes.

The paper is organized as follows. The preliminaries in Section 2 are concerned with fuzzy connectives and their algebraic properties. Section 3 reports concepts of aggregation functions together with their main properties and examples. Focusing on the median operator and the two classes of $t$-subconorm and $t$-subnorm we analyse the corresponding properties. Section 4 considers both classes, (S,N)-(sub)implications and QL-(sub)implications and their conjugate and dual constructions. The main results concerned with aggregating QL-subimplications by applying the median operator are described in Section 5. Moreover, it is shown that the median operator preserves $(\mathrm{S}, \mathrm{N})$ - and QL-implication classes. Lastly, the conclusion and final remarks are presented.

\section{FUZZY CONNECTIVES}

In the following, basic concepts of an automorphism on the unit interval $U$, fuzzy negations and fuzzy subimplications are reported, mainly according to [12, 25].

Definition 2.1. [29, Def. 0] A mapping $\rho: U \rightarrow U$ is an automorphism if it is continuous, strictly increasing and verifies the boundary conditions $\rho(0)=0$ and $\rho(1)=1$, i.e., if it is an increasing bijection on $U$.

Automorphisms are closed under composition and inverse operators. The action of an automorphism $\rho$ on $f: U^{n} \rightarrow U$, refereed as $f^{\rho}$ and called $\rho$-conjugate of $f$, is defined as

$$
f^{\rho}(\vec{x})=\rho^{-1}\left(f\left(\rho\left(x_{1}\right), \rho\left(x_{2}\right), \ldots, \rho\left(x_{n}\right)\right)\right), \forall \vec{x}=\left(x_{1}, \ldots, x_{n}\right) \in U^{n} .
$$

The family of all automorphisms is referred as $\operatorname{Aut}(U)$. 


\subsection{Fuzzy negations}

Let $U=[0,1]$ be the unit interval. A fuzzy negation (FN) $N: U \rightarrow U$ satisfies:

N1 : $N(0)=1$ and $N(1)=0 ; \quad \mathbf{N 2}:$ If $x \geq y$ then $N(x) \leq N(y), \forall x, y \in U$.

FNs satisfying the involutive property are called strong fuzzy negations (SFNs):

N3 : $N(N(x))=x, \forall x \in U$.

The standard negation $N_{S}(x)=1-x$ is a strong fuzzy negation.

Let $N$ be a FN and $f: U^{n} \rightarrow U$ be a real function. Then, for all $\vec{x}=\left(x_{1}, x_{2}, \ldots, x_{n}\right) \in U^{n}$, the $N$-dual function of $f$ is given by the expression:

$$
f_{N}(\vec{x})=N\left(f\left(N\left(x_{1}\right), N\left(x_{2}\right), \ldots, N\left(x_{n}\right)\right)\right)
$$

Notice that, when $N$ is involutive, $\left(f_{N}\right)_{N}=f$, that is the $N$-dual function of $f_{N}$ coincides with $f$. In addition, if $f=f_{N}$ then it is clear that $f$ is a self-dual function [29]. Other properties of fuzzy negations and related main extensions can be found in [5, 26, 27].

\subsection{Fuzzy subimplications}

A function $I: U^{2} \rightarrow U$ is a fuzzy (co)subimplicator if it satisfies the conditions:

I0 : $I(1,1)=I(0,1)=I(0,0)=1 ; \quad$ J0 : $J(0,0)=I(1,0)=I(1,1)=0 ;$

When a fuzzy (co)subimplicator $(J) I: U^{2} \rightarrow U$ also satisfies this boundary condition:

I1 : $I(1,0)=0 ; \quad \mathbf{J 1}: J(0,1)=1 ;$

$(J) I$ is called a fuzzy (co)implicator. And, a fuzzy ((sub)coimplicator $J$ ) (sub)implicator $I$ satisfying the properties:

I2: If $x \leq z$ then $I(x, y) \geq I(z, y) \quad \mathbf{J 2}:$ If $x \leq z$ then $J(x, y) \geq J(z, y)$ (left antitonicity);

I3 : If $y \leq z$ then $I(x, y) \leq I(x, z) \quad$ J3 : If $y \leq z$ then $J(x, y) \leq J(x, z)$ (right isotonicity);

$(J) I$ is called a (fuzzy (sub)coimplication) fuzzy (sub)implication [11, Def. 6][28]. Since a fuzzy ((sub)coimplication) (sub)implication verifies (J0) I0 and (J3) I3 then we have

I4: $I(0, y)=1 \quad \mathbf{J 4}: J(1, y)=0$ (left boundary property).

\section{AGGREGATION FUNCTIONS}

Based on [7] and [14], the general meaning of an aggregation function in FL is to assign an $n$-tuple of real numbers belonging to $U^{n}$ to a single real number on $U$, such that it is a nondecreasing and idempotent (i.e., it is the identity when an $n$-tuple is unary) function satisfying boundary conditions. In [8, Def. 2], an $n$-ary aggregation function $A: U^{n} \rightarrow U$ demands, for all $\vec{x}=\left(x_{1}, x_{2}, \ldots, x_{n}\right), \vec{y}=\left(y_{1}, y_{2}, \ldots, y_{n}\right) \in U^{n}$, the following conditions: 


\section{A1: Boundary conditions}

$$
A(\overrightarrow{0})=A(0,0, \ldots, 0)=0 \quad \text { and } \quad A(\overrightarrow{1})=A(1,1, \ldots, 1)=1 ;
$$

\section{A2: Monotonicity}

If $\vec{x} \leq \vec{y}$ then $A(\vec{x}) \leq A(\vec{y})$ where $\vec{x} \leq \vec{y}$ iff $x_{i} \leq y_{i}$, for all $0 \leq i \leq n$.

Some extra usual properties for aggregation functions are the following:

\section{A3: Symmetry}

$A\left(\overrightarrow{x_{\sigma}}\right)=A\left(x_{\sigma_{1}}, x_{\sigma_{2}}, \ldots, x_{\sigma_{n}}\right)=A(\vec{x})$, when $\sigma: \mathbb{N}^{n} \rightarrow \mathbb{N}^{n}$ is a permutation;

\section{A4: Idempotency}

$A(x, x, \ldots, x)=x$, for all $x \in U ;$

\section{A5: Continuity}

If for each $i \in\{1, \ldots, n\}, x_{1}, \ldots, x_{i-1}, x_{i+1}, \ldots x_{n} \in U$ and a convergent sequence $\left\{x_{i j}\right\}_{j \in \mathbb{N}}$ we have that:

$$
\lim _{j \rightarrow \infty} A\left(x_{1}, \ldots, x_{i-1}, x_{i j}, x_{i+1}, \ldots, x_{n}\right)=A\left(x_{1}, \ldots, x_{i-1}, \lim _{j \rightarrow \infty} x_{i j}, x_{i+1}, \ldots x_{n}\right)
$$

\section{A6: $k$-homogeneity}

For all $k \in] 0, \infty\left[\right.$ and $\alpha \in\left[0, \infty\left[\right.\right.$ such that $\alpha^{k} \vec{x}=\left(\alpha^{k} x_{1}, \alpha^{k} x_{2}, \ldots, \alpha^{k} x_{n}\right) \in U^{n}$, $A\left(\alpha^{k} \vec{x}\right)=\alpha^{k} A(\vec{x})$

A7: Distributivity of an aggregation $A: U^{n} \rightarrow U$ related to a function $F: U^{2} \rightarrow U$

$$
A\left(F\left(x, y_{1}\right), \ldots, F\left(x, y_{n}\right)\right)=F\left(x, A\left(y_{1}, \ldots, y_{n}\right)\right), \text { for all } x, y_{1}, \ldots, y_{n} \in U .
$$

\subsection{Median as a self $N_{S}$-dual operator}

In the following, the median aggregation is a self $N_{S}$-dual aggregation operator [21].

Proposition 3.1. [24, Proposition 1] For all $\vec{x} \in U^{n}$ and $\mathbf{I}=\{1,2, \ldots n\}$, let $\sigma: \mathbf{I} \rightarrow \mathbf{I}$ be a permutation function such that $x_{\sigma(i)} \leq x_{\sigma(i+1)}$, for all $i=1, \ldots n-1$. The $n$-ary aggregation function $M: U^{n} \rightarrow U$ called median aggregation and defined as follows:

$$
M(\vec{x})=\left\{\begin{array}{l}
x_{\sigma\left(\frac{n+1}{2}\right)}, n \text { is an odd number } ; \\
\frac{1}{2}\left(x_{\sigma\left(\frac{n}{2}\right)}+x_{\sigma\left(\frac{n}{2}+1\right)}\right), \text { otherwise. }
\end{array}\right.
$$

satisfies Property Ak, for $k \in\{3,4,5,6\}$.

Proposition 3.2. Let $A$ be an aggregation function and $N$ be a SFN such that:

$$
\text { N5 : } N(A(\vec{x}))=A\left(N\left(x_{1}\right), N\left(x_{2}\right), \ldots, N\left(x_{n}\right)\right) .
$$

Then we have that $A_{N}(\vec{x})=A(\vec{x}), \forall \vec{x} \in U^{n}$. 
Proof. For all $\vec{x} \in U^{n}, A_{N}(\vec{x})=N(A(N(\vec{x})))=A(N(N(\vec{x})))$.

Proposition 3.3. The median aggregation function $M$ satisfies:

$$
M_{N_{S}}(\vec{x})=M(\vec{x}), \forall \vec{x} \in U^{n} .
$$

Proof. First, when $M$ has an odd number of arguments,

$$
N_{S}(M(\vec{x}))=N_{S}\left(x_{\sigma\left(\frac{n+1}{2}\right)}\right)=M\left(N_{S}(\vec{x})\right) .
$$

Otherwise, by taking $M$ as an even number of arguments, we obtain that

$$
N_{S}(M(\vec{x}))=1-\frac{1}{2}\left(x_{\sigma\left(\frac{n}{2}\right)}+x_{\sigma\left(\frac{n}{2}+1\right)}\right)=\frac{1}{2}\left(1-x_{\sigma\left(\frac{n}{2}\right)}+1-x_{\sigma\left(\frac{n}{2}+1\right)}\right) .
$$

Thus, for all $\vec{x} \in U$, both cases state that $M$ verifies $N_{S}(M(\vec{x}))=M\left(N_{S}(\vec{x})\right)$. Therefore, by Proposition 3.2, $M_{N_{S}}(\vec{x})=M(\vec{x})$ and Eq. (3.2) is verified.

Corollary 3.1. The standard negation $N_{S}$ verifies $\mathbf{N} 5$ for median aggregation $M$.

Proposition 3.4. For each positive integer number $r$, let $\phi_{r}(x)=x^{r} \in$ Aut $(U)$. The median aggregation function $M$ verifies:

$$
M^{\phi_{r}}(\vec{x})=\left\{\begin{array}{l}
M(\vec{x}), \text { if } n \text { is odd }, \\
\sqrt[r]{\frac{1}{2}\left(x_{\sigma\left(\frac{n}{2}\right)}^{r}+x_{\sigma\left(\frac{n+1}{2}\right)}^{r}\right)}, \text { otherwise } ;
\end{array}\right.
$$

Proof. Straightforward.

\subsection{Triangular sub(co)norms}

According to [5], a triangular sub(co)norm (t-sub(co)norm) is a binary aggregation function $T: U^{2} \rightarrow U\left(S: U^{2} \rightarrow U\right)$ such that, for all $x, y \in U$, the following holds:

T0 : $T(x, y) \leq \min (x, y)$

S0 : $S(x, y) \geq \max (x, y)$

and also verifies the commutativity, associativity and monotonicity properties which are, respectively, given by the next three expressions:
T1 : $T(x, y)=T(y, x)$;
S1: $S(x, y)=S(y, x)$;
T2: $T(x, T(y, z))=T(T(x, y), z)$;
S2 : $S(x, S(y, z))=S(S(x, y), z)$;
T3 : $T(x, z) \leq T(y, z)$, if $x \leq y$;
S3 : $S(x, z) \leq S(y, z)$, if $x \leq y$.

A t-(co)norm is a t-sub(co)norm satisfying the following boundary condition:
T4: $T(x, 1)=x$;
S4 : $S(x, 0)=x$.

Remark 1. Based on Properties SO and TO, we have that: 


$$
\begin{aligned}
& S(0,0) \geq 0 ; \quad S(0,1)=1 ; \quad S(1,0)=1 ; \quad S(1,1)=1 . \\
& T(1,1) \leq 1 ; \quad T(1,0)=0 ; \quad T(0,1)=0 ; \quad T(0,0)=0 .
\end{aligned}
$$

\subsubsection{Triangular sub(co)norms and $N_{S}$-dual constructions}

In the following, the family of all t-sub(co)norms $T_{i}\left(S_{i}\right)$ is referred as $\mathcal{T}(S)$. We discuss properties of t-subnorms and t-subconorms as extensions of product and probabilistic sum in subfamilies $\mathcal{T}_{P}$ and $S_{P}$, respectively.

Proposition 3.5. For $i \geq 1$ and $x, y \in U, T_{i}\left(S_{i}\right): U^{2} \rightarrow U$ is a $t$-sub(co)norm given by

$$
T_{i}(x, y)=\frac{1}{i} x y, \quad S_{i}(x, y)=1-\frac{1}{i}(1-x-y+x y) .
$$

Proof. Straightforward.

Remark 2. Observe that, for $i=1, T_{i}$ and $S_{i}$ in Eqs.(3.4) are called the product t-norm and the probabilistic sum, respectively, and corresponding expression can be given as

$$
T_{P}(x, y)=x y, \quad S_{P}(x, y)=x+y-x y .
$$

Additionally, each pair $\left(T_{i}, S_{i}\right) \in \mathcal{T} \times S$ defines a pair of $N_{S}$-mutual dual functions. That means, by Eq. (2.2), both equations $\left(T_{i}\right)_{N_{S}}=S_{i}$ and $\left(S_{i}\right)_{N_{S}}=T_{i}$ are verified, therefore $T_{i}$ and its $N_{S}$-dual construction $S_{i}$ is a pair of $N_{S}$-mutual dual functions, for $i \geq 1$.

Proposition 3.6. For $i \geq 1, T_{i}: U^{2} \rightarrow U\left(S_{i}: U^{2} \rightarrow U\right)$ is a $t$-sub(co)norm satisfying

T5 : $T_{i}\left(x, N_{S}(x)\right)=0$ iff $x=0$ or $x=1 ; \quad$ S5 : $S_{i}\left(x, N_{S}(x)\right)=1$ iff $x=0$ or $x=1$;

Proof. For all $x, y \in U$, we have that

$$
\begin{aligned}
& T_{i}\left(x, N_{S}(x)\right)=0 \quad \Leftrightarrow \quad \frac{1}{i}\left(x-x^{2}\right)=0 \Leftrightarrow x-x^{2}=0 \Leftrightarrow x=0 \text { or } x=1 ; \\
& S_{i}\left(x, N_{S}(x)\right)=1 \quad \Leftrightarrow \quad 1-\frac{1}{i}(x(1-x))=1 \Leftrightarrow x-x^{2}=0 \Leftrightarrow x=0 \text { or } x=1 .
\end{aligned}
$$

Therefore, $T_{i}$ and $S_{i}$ satisfy Properties $\mathbf{T 5}$ and $\mathbf{S 5}$, respectively.

\subsubsection{Extending triangular sub(co)norms to triangular (co)norms}

In the following proposition, we can obtain $T_{P}$ from t-(co)norms $T_{i}\left(S_{i}\right)$.

Proposition 3.7. For all $x, y \in U$, for each index $i$ such that $i \geq 1$, the following holds:

$$
T_{P}(x, y)=i T_{i}(x, y), \quad S_{P}(x, y)=1-i\left(1-\left(S_{i}(x, y)\right)\right),
$$


Proof. Straightforward.

By applying the results of Proposition 2.4 in [5], we obtain a t-(co)norm $T_{T_{i}}\left(S_{S_{i}}\right.$ ) from a tsub(co)norm $T_{I} i\left(S_{i}\right)$ as presented in the following:

Corollary 3.2. Let $T_{i}\left(S_{i}\right): U^{2} \rightarrow U$ be the $t$-sub(co)norm defined in Eq. (3.4). Then the function $T_{\left(T_{i}, \min \right)},\left(S_{\left(S_{i}, \min \right)}\right): U^{2} \rightarrow U$ given as

$$
\begin{aligned}
& T_{\left(T_{i}, \min \right)}(x, y)=\left\{\begin{array}{l}
T_{i}(x, y), \text { if }(x, y) \in\left[0,1\left[^{2},\right.\right. \\
\min (x, y), \text { otherwise } ;
\end{array}\right. \\
& S_{\left(S_{i}, \min \right)}(x, y)=\left\{\begin{array}{l}
S_{i}(x, y), \text { if }(x, y) \in\left[0,1\left[^{2},\right.\right. \\
\max (x, y), \text { otherwise } ;
\end{array}\right.
\end{aligned}
$$

is a $t$-(co)norm.

Proof. Straightforward.

\subsubsection{Conjugate Triangular Sub(co)norms}

Proposition 3.8. Consider $\phi_{r}(x)=x^{r}, \psi_{r}(x)=1-(1-x)^{r}$ in Aut $(U)$ defined by Proposition 3.8. Then, the following holds:

$$
T_{i}^{\phi_{r}}(x, y)=T_{\phi_{r}^{-1}(i)}(x, y), \quad \text { and } \quad S_{i}^{\psi_{r}}(x, y)=S_{\phi_{r}{ }^{-1}(i)}(x, y), \forall x, y \in U .
$$

Proof. For all $i \geq 1$ and $x, y \in U$, we have that:

$$
\begin{aligned}
T_{i}^{\phi_{r}}(x, y) & =\phi_{r}^{-1}\left(\frac{1}{i}\left(\phi_{r}(x) \phi_{r}(y)\right)\right. \\
& =\phi_{r}^{-1}\left(\frac{1}{i} x^{r} y^{r}\right) \\
& =\frac{1}{\phi_{r}^{-1}(i)} x y ; \\
& =T_{\phi_{r}}^{-1}(i)(x, y) ; \\
S_{i}^{\psi_{r}}(x, y) & =\psi_{r}^{-1}\left(1-\frac{1}{i}\left(1-\psi_{r}(x)\right)\left(1-\psi_{r}(y)\right)\right) \\
& =\psi_{r}^{-1}\left(1-\frac{1}{i}(1-x)^{r}(1-y)^{r}\right) \\
& =1-\sqrt[r]{\frac{1}{i}(1-x)^{r}(1-y)^{r}} \\
& =1-\frac{1}{\phi_{r}^{-1}(i)}(1-x)(1-y) \\
& =S_{\phi_{r}^{-1}(i)}(x, y) .
\end{aligned}
$$


Therefore, $T_{i}^{\phi_{r}} \in \mathcal{T}$ and $S_{i}^{\phi_{r}} \in S$.

Remark 3. Based on Proposition 3.8, if $\phi_{2}(x)=x^{2}$ and $\psi_{2}(x)=1-(1-x)^{2}$ we have that

$$
T_{i}^{\phi_{2}}=\frac{1}{\sqrt{i}} x y=T_{\sqrt{i}}(x, y) \in \mathcal{T} \quad \text { and } \quad S_{i}^{\psi_{2}}=1-\frac{1}{\sqrt{i}}(1-x)(1-y)=S_{\sqrt{i}}(x, y) \in S .
$$

However, it is not true when we permute the automorphism, which means

$$
T_{i}^{\psi_{2}}(x, y)=1-\sqrt{(1-y)^{2}+y^{r}(1-x)^{2}} \quad \text { and } \quad S_{i}^{\phi_{2}}(x, y)=\sqrt{1-\frac{1}{i}\left(1-x^{2}\right)\left(1-y^{2}\right)} .
$$

Therefore, $T_{i}^{\psi_{2}}$ and $S_{i}^{\phi_{2}}$ can not be expressed as members of $\mathcal{T}$ and $S$, respectively.

\section{4 (S,N)- AND QL-(SUB)IMPLICATION CLASSES}

The main results considered in this section were studied in [29] and [30].

\subsection{Fuzzy (S,N)-subimplications and dual construction}

An (S,N)-subimplicator is a subimplicator derived from a t-subconorm $S$ and a FN $N$. Exploring other properties such as exchange principle and contraposition, a subclass of connectives called $(\mathrm{S}, \mathrm{N})$-subimplications are studied, see details in [22].

A function $I_{S, N}\left(J_{S, N}\right): U^{2} \rightarrow U$ is called an (S,N)-subimplication ((T,N)-subcoimplication) if there exists a t-subconorm $S$ (t-norm $T$ ) and a fuzzy negation $N$ such that

$$
I_{S, N}(x, y)=S(N(x), y), \quad \text { and } \quad J_{T, N}(x, y)=T(N(x), y), \forall x, y \in U
$$

for all $x, y \in U$. If $N$ is a strong $\mathrm{FN}$, then we denote $I_{S}\left(J_{T}\right)$ and call it an $\mathbf{S}$-subimplication (T-subcoimplication).

Proposition 4.1. [22, Prop. 4.10] The following statements are equivalent:

1. $I: U^{2} \rightarrow U$ is an $(S, N)$-implication along with the underlying continuous $F N N$ and a $t$-subconorm $S$ at point 0 ;

2. $I: U^{2} \rightarrow U$ is continuous at point $x=1$ in the first component, satisfying $\mathbf{I} 3$ and the two additional conditions:

I5 : Exchange Principle : $I(x, I(y, z))=I(y, I(x, z))$, for all $x, y, z \in U$;

I6 : Contrapositive Symmetry : $I(x, y)=I(N(y), N(x))$, for all $x, y \in U$.

Proposition 4.2. An (S,N)-sub(co)implicator is a sub(co)implicator. 
Proof. By Property I0, we have that $I_{S, N}(0,0)=S(N(0), 0)=S(1,0)=1 ; I_{S, N}(1,1)=$ $S(N(1), 1)=S(0,1)=1$; and $I_{S, N}(0,1)=S(N(0), 1)=S(0,1)=1$.

Clearly, a fuzzy (co)implication $I_{S, N}\left(J_{S, N}\right)$ is also a fuzzy sub(co)implication. The family of all (S,N)-subimplications ((T,N)-subcoimplications) is denoted as $\mathfrak{I}_{S}\left(\mathfrak{I}_{T}\right)$. Additionally, if $S=T_{N}$, the $N$-dual function of an subimplication $I_{S, N}$ is a subcoimplication $J_{S_{N}, N}$, meaning that $J_{S_{N}, N}=\left(I_{S, N}\right)_{N}$.

Since the dual construction of Proposition 4.1 is also satisfied, the following holds:

Proposition 4.3. For all $x, y \in U$, the binary function $I_{i},\left(J_{i}\right): U^{2} \rightarrow U$, defined as

$$
I_{i}(x, y)=1-\frac{1}{i}(x-x y) \quad \text { and } \quad J_{i}(x, y)=\frac{1}{i}(y-x y),
$$

is a fuzzy (S,N)-sub(co)implicator.

Proof. I0 is immediate. Additionally, for all $x, y \in U$, by taking $S_{i}(x, y)=1-\frac{1}{i}(1-x-y+$ $x y$ ), for $i \geq 1$, we have that

$$
S_{i}\left(N_{S}(x), y\right)=1-\frac{1}{i}(1-(1-x)-y+(1-x) y)=1-\frac{1}{i}(x-x y) .
$$

Consequently, $I_{i}(x, y)=S_{i}\left(N_{S}(x), y\right)$. Therefore $I_{i}$ is an $\left(S_{i}, N_{S}\right)$-implicator. Analogously, subcoimplication $J_{i}$ is also proved.

Proposition 4.4. [24, Prop. 5] An (S,N)-subimplication satisfies Property $\mathbf{I}_{\mathbf{k}}$, for $\mathbf{k} \in\{0$, $2,3,4,5,6\}$.

\subsubsection{Extending (S,N)-subimplications to (S,N)-implications}

See in Figure 1 a graphical representation for three examples of subimplications, $I_{1}, I_{2}, I_{3} \in \mathfrak{\Im}_{S}$. In particular, $I_{1}$ is referred to the Reichenbach's implication and denoted as $I_{R H}$.
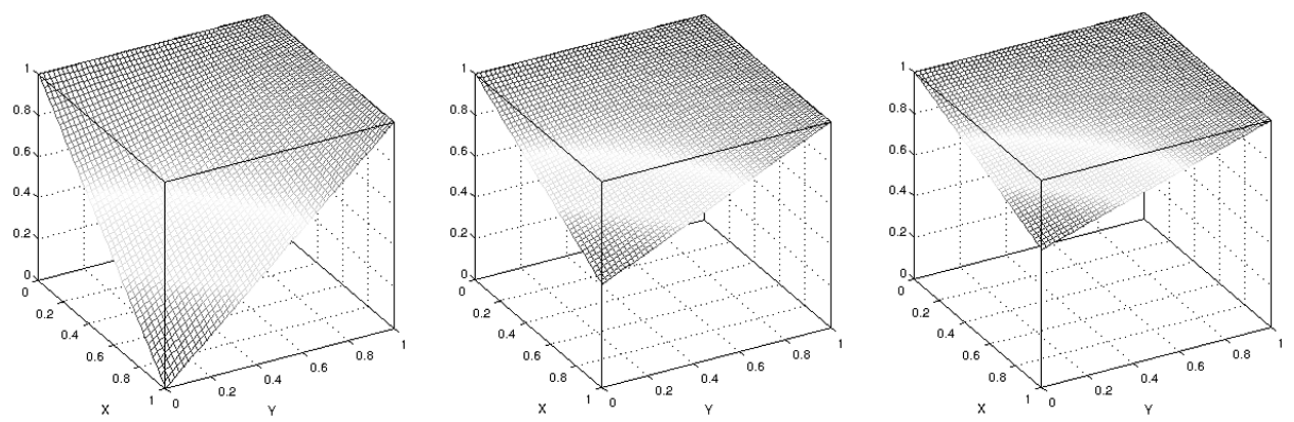

Figure 1: Fuzzy (S,N)-subimplications of family $\mathfrak{\Im}=\left\{I_{1}, I_{2}, I_{3}\right\}$. 
In the following, we discuss the extension of an $\left(S_{i}, N_{S}\right)$-subimplication $I_{i}$ to an $\left(S_{i}, N_{S}\right)$ implication. Based on the duality stated by $N_{S}$ in the class $\mathfrak{\Im}_{T}$, the extension of an $\left(T_{i}, N_{S}\right)$ subcoimplication to an $\left(T_{i}, N_{S}\right)$-implication can also be obtained, analogously.

As a consequence, from Proposition 4.5, we can obtain the Reichenbach's implication $I_{R H}$ from each $U_{I}$-extended member $I_{i}$ of family $\mathfrak{S}$. Additionally, its related $N_{S}$-dual construction, the Reichenbach's coimplication $J_{R H}$, can also be obtained.

Proposition 4.5. Let $i$ be an index such that $i \geq 1$. Then, for all $x, y, \in U$, we have that

$$
I_{R H}(x, y)=1-i\left(1-I_{i}(x, y)\right) \quad \text { and } \quad J_{R H}(x, y)=i\left(J_{i}(x, y)\right) .
$$

Proof. Straightforward.

Proposition 4.6. Let $I_{i}\left(J_{i}\right): U^{2} \rightarrow U$ be the $(S, N)$-sub(co)implication defined in Proposition 4.3, by Eq. (4.2). Then the function defined as

$$
\begin{aligned}
& I_{S i}(x, y)= \begin{cases}0, & \text { if } x=1 \text { and } y=0, \\
I_{i}(x, y), & \text { otherwise } ;\end{cases} \\
& J_{T i}(x, y)= \begin{cases}1, & \text { if } x=0 \text { and } y=1, \\
J_{i}(x, y), & \text { otherwise; }\end{cases}
\end{aligned}
$$

is an (co)implication.

Proof. Property I1 is immediate. Moreover, it follows from Proposition 4.4 that $I_{S_{i}}$ also verifies $\mathbf{I 0}$ and properties from I2 to I4. $J_{T i}$ is also proved. Proposition 4.6 holds.

Based on the results of Proposition 4.6, $(\mathrm{S}, \mathrm{N})$-sub(co)implications can be extended in order to obtain (co)implications. In particular, $I_{1}=I_{S 1}=I_{R H} \in \mathfrak{\Im}_{S}$.

\subsubsection{Conjugate (S,N)-subimplications}

In the following, the action of an automorphism on $U$ is discussed.

Proposition 4.7. Consider $\phi_{r}(x)=x^{r}, \psi_{r}(x)=1-(1-x)^{r}$ in Aut $(U)$ defined by Proposition 3.8. Then, for all $x, y \in U$, the following holds:

$$
\begin{gathered}
\left(I_{S_{i}, N_{S}}\right)^{\psi_{r}}(x, y)=I_{\psi_{\psi_{r}-1}(i), N_{S}^{\psi_{r}}}(x, y) \\
\text { and } \\
\left(J_{T_{i}, N_{S}}\right)^{\phi_{r}}(x, y)=J_{\phi_{\phi_{r}-1}(i), N_{S}^{\phi_{r}}}(x, y)
\end{gathered}
$$


Proof. For all $i \geq 1$ and $x, y \in U$, by Eqs. (2.1)(4.1) and (3.4), we have that:

$$
\begin{aligned}
\left(I_{S_{i}, N_{S}}\right)^{\psi_{r}}(x, y) & =\psi_{r}^{-1}\left(I_{S_{i}, N_{S}}\left(\psi_{r}(x), \psi_{r}(y)\right)\right) \\
& =\psi_{r}^{-1}\left(S_{i}\left(N_{S} \circ \psi_{r}(x), \psi_{r}(y)\right)\right) \\
& =S_{i}^{\psi_{r}}\left(\left(\psi_{r}^{-1} \circ N_{S} \circ \psi_{r}\right)(x), y\right) \\
& =S_{i}^{\psi_{r}}\left(N_{S}^{\psi_{r}}(x), y\right)=I_{S_{i}^{\psi_{r}}, N_{S}^{\psi_{r}}}(x, y)
\end{aligned}
$$

Additionally, by Eq. (3.7) in Proposition 3.8, $\left(I_{S_{i}, N_{S}}\right)^{\psi_{r}}(x, y)=I_{S_{\psi_{r}-1}(i), N_{S}^{\phi_{r}}}(x, y)$. Analogously, the dual construction can be proved. Therefore, Proposition 4.7 is verified.

\subsection{Fuzzy QL-(sub)implication class}

Fuzzy QL-(sub)implicators are reviewed. See [27, 31] and [25] for additional information.

Definition 4.1. Let $N$ be a fuzzy negation. A function $I_{S, T, N}: U^{2} \rightarrow U$ is called a $Q \mathbf{L}$ sub(co)implicator if, for $x, y \in U$, there exist a $t$-subconorm $S$ ( $t$-subnorm $T$ ) and a $t$-subnorm $T$ (t-subconorm $S$ ) such that:

$$
I_{S, N, T}(x, y)=S(N(x), T(x, y)), \quad \text { and } \quad J_{T, N, S}(x, y)=T(N(x), S(x, y)) .
$$

Proposition 4.8. A QL-subimplicator is a subimplicator.

Proof. By Property I0, we have that $I_{S, N, T}(0,0)=S(N(0), T(0,0))=S(1,0)=1$; $I_{S, N, T}(1,1)=S(N(1), T(1,1))=S(0,1)=1$; and $I_{S, N, T}(0,1)=S(N(0), T(0,1))=$ $S(1,0)=1$.

Thus, a QL-subimplicator $I_{S, N, T}$ generates the underlying t-subconorm, negation and t-norm as $S, N$ and $T$, respectively. Analogously, we can obtain to QL-subcoimplicator $I_{T, N, S}$. The family of all fuzzy QL-sub(co)implicators is referred as $\mathfrak{s}_{Q L}\left(\mathcal{J}_{Q L}\right)$.

Proposition 4.9. For all $x, y \in U$, the function $I_{Q L_{i}}\left(J_{Q L_{i}}\right): U^{2} \rightarrow U$, given by

$$
I_{Q L_{i}}(x, y)=1-\frac{1}{i}\left(x-x^{2} y\right) \quad \text { and } \quad\left(J_{Q L_{i}}(x, y)=\frac{1}{i}(1-x)(x+y+x y)\right),
$$

is a fuzzy $Q L-s u b($ co)implicator.

Proof. For all $x, y \in U$, we have that

$$
\begin{aligned}
I_{S_{i}, N_{S}, T_{P}}(x, y) & =S_{i}\left(N_{S}(x), T(x, y)\right)=1-\frac{1}{i}(1-(1-x)-x y+(1-x) x y) \\
& =1-\frac{1}{i}\left(x-x^{2} y\right)=I_{Q L_{i}}(x, y) .
\end{aligned}
$$

This means that, $I_{Q L_{i}} \in \mathfrak{I}_{Q L}$. Analogously, we can prove that $J_{Q L_{i}} \in \mathcal{J}_{Q L}$. 
The following proposition is an extension of Proposition 4.2 in [27] by considering the main algebraic properties that characterize the fuzzy QL-subimplication class.

Proposition 4.10. A QL-subimplicator $I_{S, T, N} \in \mathfrak{S}_{Q L}$ satisfies $\mathbf{I k}$ for $\mathbf{k} \in\{0,2,4\}$ together with the additional property:

$$
\text { I9: if } x_{1} \geq x_{2} \text { then } I\left(x_{1}, 0\right) \leq I\left(x_{2}, 0\right) \text {, for all } x_{1}, x_{2} \in U \text {. }
$$

In addition, when $T(S): U^{2} \rightarrow U$ is a $t$-(co)norm the following holds:

$$
\text { I10(a) : I }(1, y) \geq y, \text { for all } y \in U ; \quad \text { and } \mathbf{I 1 0}(\mathbf{b}): I(1, y) \leq y, \text { for all } y \in U \text {. }
$$

Proof. For $x_{1}, x_{2}, x, y_{1}, y_{2}, y \in U, \mathbf{I} \mathbf{0}$ is immediate. The following is verified:

I2 Since $S$ and $T$ are monotonic functions, if $y_{1} \leq y_{2}$ then $T\left(x, y_{1}\right) \leq T\left(x, y_{2}\right)$ and consequently, $I_{S, N, T}\left(x, y_{1}\right)=S\left(N(x), T\left(x, y_{1}\right)\right) \leq S\left(N(x), T\left(x, y_{2}\right)\right)=I_{S, N, T}\left(x, y_{2}\right)$.

I4 $I_{S, N, T}(0, y)=S(1, T(0, y))=S(1,0)=1$.

19 When $x \geq y$ then $N(x) \leq N(y)$. Then, $I_{S, N, T}(x, 0)=S(N(x), T(x, 0))=S(N(x), 0) \leq$ $S(N(y), 0)=S(N(y), T(y, 0))=I_{S, N, T}(y, 0)$.

I10(a) $I_{S, N, T}(1, y)=S(0, T(1, y))=S(0, y) \geq y$;

I10(a) $I_{S, N, T}(1, y)=S(0, T(1, y))=T(1, y) \leq y$.

Therefore, Proposition 4.10 is verified.

Corollary 4.3. The operator $I_{S_{P I}, N_{S}, T_{P}} \in \mathfrak{\Im}$ verifies $\boldsymbol{I} \boldsymbol{k}$ for $\mathbf{k} \in\{0,2,4,9,10\}$.

Proof. Straightforward from Proposition 4.10.

Remark 4. Let I : $U^{2} \rightarrow U$ be a function given by Eq.(4.4). By taking a $t$-subconorm $S$, a fuzzy negation $N$ and a t-subnorm $T$, the function I does not satisfy either $\mathbf{I 0}$ or I1:

$$
I(1,1)=S(N(1), T(1,1)) \geq T(1,1) ; \text { and } I(1,0)=S(N(1), T(1,0))=S(0,0) \geq 0 .
$$

Therefore, I is not necessarily a subimplicator.

\subsubsection{Extending QL-subimplicators to QL-implicators}

Clearly, a QL-implicator is always a QL-subimplicator. In Figure 2, instances $I_{Q L_{1}}, I_{Q L_{2}}$ and $I_{Q L_{3}}$ of such class $\mathfrak{\Im}_{Q L}$ are graphically presented. In particular, $I_{Q L_{1}} \in \Im$ is a QL-implicator [30] 

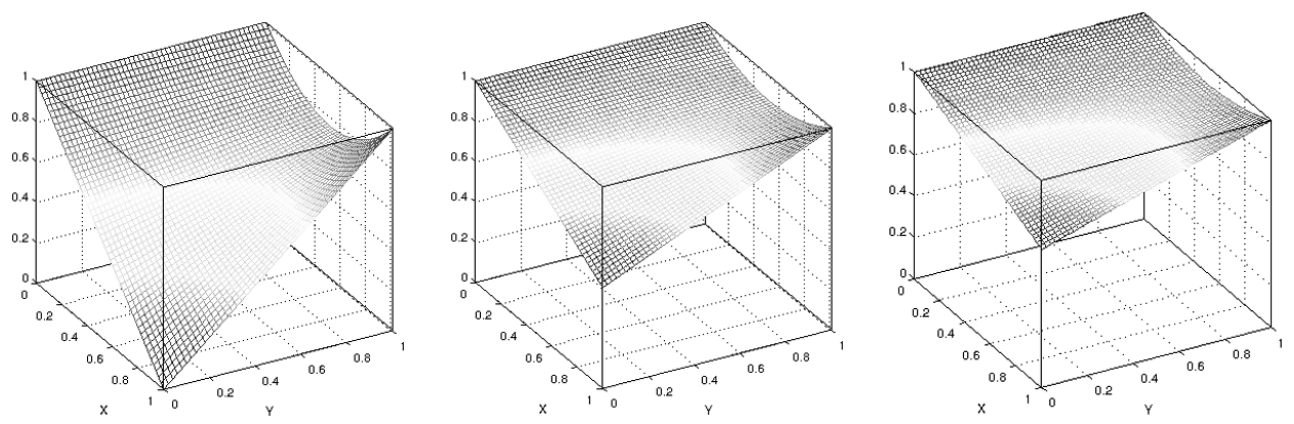

Figure 2: Fuzzy QL-subimplications of family $\mathfrak{\Im}_{Q L}=\left\{I_{Q L 1}, I_{Q L 2}, I_{Q L 3}\right\}$.

and $I_{Q L_{2}}$ and $I_{Q L_{3}}$ can be transformed into $I_{Q L_{1}}$ based on results of Proposition 4.3. This section also discusses a (converse) construction in the class $\mathfrak{\Im}_{Q L}\left(\mathcal{J}_{Q L}\right)$, by considering the main conditions under which a QL-sub(co)implicator can be extended to a QL-(co)implicator, see Proposition 4.12.

Proposition 4.11. For each index $i$ such that $i \geq 1$ and $x, y \in U$, the following holds:

$$
\left.I_{Q L 1}(x, y)=i\left(1-I_{Q L_{i}}(x, y)\right) \quad \text { and } \quad J_{Q L 1}(x, y)=i J_{Q L_{i}}(x, y)\right) .
$$

Proof. Straightforward.

Proposition 4.12. Let $I_{S_{i}, N_{S}, T_{P}}\left(J_{S_{i}, N_{S}, T_{P}}\right): U^{2} \rightarrow U$ be the $Q L$-sub(co)implication defined in Proposition 4.11, by Eq. (4.6). Then the function defined as

$$
\begin{aligned}
& I_{I_{S_{i}, N_{S}, T_{P}}}(x, y)=\left\{\begin{array}{l}
0, \text { if } x=1 \text { and } y=0, \\
I_{S_{i}, N_{S}, T_{P}}(x, y), \text { otherwise }
\end{array}\right. \\
& J_{J_{S_{i}, N_{S}, T_{P}}}(x, y)=\left\{\begin{array}{l}
1, \text { if } x=0 \text { and } y=1, \\
J_{S_{i}, N_{S}, T_{P}}(x, y), \text { otherwise }
\end{array}\right.
\end{aligned}
$$

is a $Q L-($ co)implication.

Proof. It follows from Propositions 4.9 and 3.2 and Definition 4.1.

\subsubsection{Conjugate QL-subimplicators}

In the following, an automorphism $\psi(\phi) \in A u t(U)$ is considered in order to obtain conjugate functions in the class of QL-sub(co)implications $\mathfrak{\Im}_{Q L}\left(\mathcal{J}_{Q L}\right)$. 
Proposition 4.13. Consider $\phi_{r}(x)=x^{r}, \psi_{r}(x)=1-(1-x)^{r}$ in Aut $(U)$ defined by Proposition 3.8. Then, the following holds:

$$
\begin{aligned}
& \left(I_{S_{i}, N_{S}, T_{P}}\right)^{\psi_{r}}(x, y)=I_{S_{\phi_{r}-1}(i)}, N_{S}^{\psi_{r}}, T_{P}^{\psi_{r}}(x, y) \text { and } \\
& \left(J_{S_{P}, N_{S}, T_{i}}\right)^{\phi_{r}}(x, y)=J_{S_{P}, N_{S}, T_{\phi_{r}^{-1}(i)}}(x, y) .
\end{aligned}
$$

Proof. For all $i \geq 1$ and $x, y \in U$, we have that:

$$
\begin{aligned}
\left(I_{S_{i}, N_{S}, T_{P}}\right)^{\psi_{r}}(x, y) & =\psi_{r}^{-1}\left(S_{i}\left(N_{S}(\psi(x)), T_{P}\left(\psi_{r}(x), \psi(y)\right)\right)\right. \\
& =\psi_{r}^{-1}\left(S_{i}\left(\left(\psi_{r} \circ \psi_{r}^{-1}\right) N_{S}\left(\psi_{r}(x)\right),\left(\psi_{r} \circ \psi_{r}{ }^{-1}\right) T_{P}\left(\psi_{r}(x), \psi_{r}(y)\right)\right)\right. \\
& =\left(S_{i}^{\phi_{r}}\left(N_{S}^{\phi_{r}}(x), T_{P}^{\phi_{r}}(x, y)\right)\right. \\
& =I_{S^{\psi_{r}}, T^{\psi_{r}}, N^{\psi_{r}}}(x, y) \\
& =I_{S_{\phi_{r}-1}(i)}, N_{S}^{\psi_{r}}, T_{P}^{\psi_{r}}(x, y) .
\end{aligned}
$$

Therefore, $I_{i}^{\psi_{r}} \in \mathfrak{\Im}_{Q L}$. Analogously, it can be proved that $J_{i}^{\phi_{r}} \in \mathfrak{I}_{Q L}$.

\section{AGGREGATING CONNECTIVES FROM THE MEDIAN OPERATOR}

Consider $A: U^{n} \rightarrow U$ as an $n$-ary aggregation function and $\mathcal{F}=\left\{F_{i}: U^{2} \rightarrow U\right\}$, with $i \in$ $\{1,2, \ldots, n\}$ as a family of $n$-ary functions in the following results of this section.

Definition 5.1. [22, Proposition 5.1] An k-ary function $\mathcal{F}_{A}: U^{k} \rightarrow U$ is called an $(A, \mathcal{F})$ operator on $U$ and is given by:

$$
\mathcal{F}_{A}\left(x_{1}, \ldots, x_{k}\right)=A\left(F_{1}\left(x_{1}, \ldots, x_{k}\right), \ldots, F_{n}\left(x_{1}, \ldots, x_{k}\right)\right) .
$$

\subsection{Aggregating fuzzy t-sub(co)norms}

The conditions under which a class of t-sub(co)norms is preserved by the median operator are discussed. Additionally, conjugate and dual constructions related to the family of t-sub(co)norms $(S) \mathcal{T}=\left\{\left(S_{i}\right) T_{i}: U^{2} \rightarrow U\right\}$, with $i \in \mathbf{I}=\{1,2, \ldots, n\}$ are also analyzed.

Proposition 5.1. [22, Proposition 6.1] Let $A: U^{n} \rightarrow U$ be an aggregation function and $(S) \mathcal{T}=$ $\left\{\left(S_{i}\right) T_{i}: U^{2} \rightarrow U\right\}$, with $i \in\{1,2, \ldots, n\}$ be a family of $t$-sub(co)norms. Then the function $\left(S_{A}: U^{2} \rightarrow U\right) \mathcal{T}_{A}: U^{2} \rightarrow U$, called $((A, S)$-operator $)(A, \mathcal{T})$-operator, is a $t$-sub(co)norm whenever the following two conditions are satisfied:

(i) A satisfies property $\mathbf{A} 7$; and

(ii) each $t$-sub(co)norm $\left(S_{i}\right) T_{i}$ satisfies the generalized associativity ${ }^{3}$ :

$$
\begin{aligned}
& S_{i}\left(x, S_{j}(y, z)\right)=S_{i}\left(S_{j}(x, y), z\right) ; \\
& T_{i}\left(x, T_{j}(y, z)\right)=T_{i}\left(T_{j}(x, y), z\right), \forall i \in \mathbf{I}, \forall x, y, z \in U
\end{aligned}
$$

${ }^{3}$ Eq. (5.2) are particular cases of Eq. (GA) in [32].

Tend. Mat. Apl. Comput., 16, N. 3 (2015) 
Proposition 5.2. Let $\mathcal{T}$ and $S$ be the families of $t$-subnorms and $t$-subconorms described in Proposition 3.5. For all $i, j \geq 1$, each pair $T_{i}, T_{j} \in \mathcal{T}$ and $S_{i}, S_{j} \in S$ satisfies Eqs. (5.2)a and (5.2) b, respectively.

Proof. For all $x, y, z \in U, T_{i}\left(x, T_{j}(y, z)\right)=T_{i}\left(x, \frac{1}{j} y z\right)=\frac{1}{i j}(x y z)=\frac{1}{i}\left(T_{j}(x, y) \cdot z\right)=$ $T_{i}\left(T_{j}(x, y), z\right)$ Then, $\mathcal{T}$ satisfies the Eq. (5.2)a. The proof for $S$ and related to Eq. (5.2) can be analogously obtained.

Proposition 5.3. [24, Proposition 10] Let $\sigma: \mathbf{I} \rightarrow \mathbf{I}$ be a permutation (Proposition 3.1) together with $\mathcal{T}$ and $S$ be the corresponding families, such that for all $x, y \in U$, it holds:

$$
\begin{aligned}
\mathcal{T} & =\left\{T_{i}(x, y)=\frac{1}{i} x y: i \in \mathbf{I}\right\} \quad \text { and } \\
\mathcal{S} & =\left\{S_{i}(x, y)=1-\frac{1}{i}(1-x-y+x y): i \in \mathbf{I}\right\} .
\end{aligned}
$$

According to Eq. (5.1), for all $x, y \in U$, the operators $\mathcal{T}_{M}, S_{M}: U^{2} \rightarrow U$ given by

$$
\begin{aligned}
& (\mathcal{T})_{M}(x, y)=\left\{\begin{array}{l}
\frac{1}{\sigma\left(\frac{n+1}{2}\right)} x y, \text { if } n \text { is odd, } \\
\left(\frac{1}{2 \sigma\left(\frac{n}{2}\right)}+\frac{1}{2 \sigma\left(\frac{n+1}{2}\right)}\right) x y, \text { otherwise }
\end{array}\right. \\
& (S)_{M}(x, y)=\left\{\begin{array}{l}
1-\frac{1}{2 \sigma\left(\frac{n+1}{2}\right)}(1-x)(1-y), \text { if } n \text { is odd } \\
1-\left(\frac{1}{2 \sigma\left(\frac{n}{2}\right)}+\frac{1}{2 \sigma\left(\frac{n+1}{2}\right)}\right)(1-x)(1-y), \text { otherwise }
\end{array}\right.
\end{aligned}
$$

respectively, satisfy Property $\mathbf{A} 7$.

Proof. For all $x, y \in U$, consider the following two distinct cases.

(i) First, if $n$ is odd, we obtain the following:

$$
\begin{aligned}
(\mathcal{T})_{M}(x, y) & =M\left(T_{1}(x, y), \ldots, T_{n}(x, y)\right) \\
& =\frac{1}{\sigma\left(\frac{n+1}{2}\right)} x y \\
& =T_{\sigma\left(\frac{n+1}{2}\right)}(x, y) \\
& =T_{\sigma\left(\frac{n+1}{2}\right)}(x, M(y, \ldots, y)) .
\end{aligned}
$$


(ii) Otherwise, when $n$ is even, we have that:

$$
\begin{aligned}
(\mathcal{T})_{M}(x, y) & =\left(\frac{1}{2 \sigma\left(\frac{n}{2}\right)}+\frac{1}{2 \sigma\left(\frac{n+1}{2}\right)}\right) x y \\
& =T\left(\frac{1}{\sigma\left(\frac{n}{2}\right)}+\frac{1}{\sigma\left(\frac{n+1}{2}\right)}\right)^{(x, y)} \\
& =T\left(\frac{1}{\sigma\left(\frac{n}{2}\right)}+\frac{1}{\sigma\left(\frac{n+1}{2}\right)}\right)^{(x, M(y, \ldots, y)) .}
\end{aligned}
$$

Therefore $\mathcal{T}_{M}$ verifies A7. The proof related to $S_{M}$ can be analogously obtained.

Corollary 5.4. The operator $\left((S)_{M}\right)(\mathcal{T})_{M}$ is a $t$-sub(co)norm.

Proof. Straightforward from Propositions 5.1, 5.2 and 5.3.

The following proposition, reported in [22], states the conditions under which a fuzzy subimplication $I_{M}$ satisfies the exchange principle.

Proposition 5.4. [22, Proposition 5.5] Let $A: U^{n} \rightarrow U$ be an n-ary aggregation and $\mathcal{I}=$ $\left\{I_{i}: U^{2} \rightarrow U\right\}$, for $i \in \mathbf{I}=\{1,2, \ldots, n\}$ be a family of fuzzy subimplication functions. $\mathcal{I}_{A}$ satisfies $\mathbf{I}$ when the aggregation A verifies $\mathbf{A} 7$ and $\mathcal{I}$ satisfies the following property:

I10: Generalized Exchange Principle: $\forall x, y, z \in U$ and $I_{i}, I_{j} \in \mathcal{I}$, such that $0 \leq i, j \leq n,{ }^{4}$

$$
I_{i}\left(x, I_{j}(y, z)\right)=I_{i}\left(y, I_{j}(x, z)\right) .
$$

Proposition 5.5. $\mathcal{T}_{M}\left(S_{M}\right): U^{2} \rightarrow U$ is a $t$-sub(co)norm satisfying $\mathbf{T 5}$ (S5).

Proof. For all $x, y \in U$, we have that

$$
(\mathcal{T})_{M}(x, N(x))=1 \Leftrightarrow\left\{\begin{array}{l}
\frac{1}{\sigma\left(\frac{n+1}{2}\right)} x(1-x)=1 \Leftrightarrow x=0 \text { or } \\
x=1, \text { if } n \text { is odd, } \\
\left(\frac{1}{2 \sigma\left(\frac{n}{2}\right)}+\frac{1}{2 \sigma\left(\frac{n+1}{2}\right)}\right) x(1-x)=1 \Leftrightarrow x=0 \text { or } \\
x=1, \text { otherwise; }
\end{array}\right.
$$

Then, Property $\mathbf{T 5}$ is verified by $\mathcal{T}_{M}$. Analogously, its dual construction can be proven.

${ }^{4}$ This property also can be considered as a generalization of the extended migrative property, see [33, Def. 2]. 


\subsubsection{Conjugate and dual t-subnorms obtained by median aggregation}

The aim of this section is to study in more detail the interrelations between the classes of aggregated t-(co)norms and their possible conjugate functions. Another interesting issue is to study how the method can take into account their dual constructions, the classes of (T,N)subimplications $[34,35]$. It is interesting to obtain new connectives preserving the main properties in the fuzzy connective classes.

Proposition 5.6. Consider $N_{S}$ which verifies $\mathbf{N 5}$ for the median $M$. When $\left(T_{i}, S_{i}\right) \in S \times S$ is a pair of mutual $N_{S}$-dual functions on $U$, the following holds:

$$
\left(S_{M}\right)_{N_{S}}(x, y)=(\mathcal{T})_{M}(x, y) \quad \text { and } \quad\left(\mathcal{T}_{M}\right)_{N_{S}}(x, y)=(S)_{M}(x, y), \forall x, y \in U
$$

Proof. For $x, y \in U$, by Eq. (2.2), we have that $\left(S_{M}\right)_{N_{S}}(x, y)=N_{S}\left(S_{M}\left(N_{S}(x), N_{S}(y)\right)\right)$. So,

$$
\begin{aligned}
\left(S_{M}\right)_{N_{S}}(x, y) & =N_{S}\left(M\left(S_{1}\left(N_{S}(x), N_{S}(y)\right)\right), \ldots,\left(S_{n}\left(N_{S}(x), N_{S}(y)\right)\right)\right) \text { by Eq. (5.5) } \\
& \left.=M\left(N_{S}\left(S_{1}\left(N_{S}(x), N_{S}(y)\right)\right), \ldots, N_{S}\left(S_{n}\left(N_{S}(x), N_{S}(y)\right)\right)\right)\right) \text { by Eq. (3.2) }
\end{aligned}
$$

Therefore, $\left(S_{M}\right)_{N_{S}}(x, y)=M\left(T_{1}(x, y), \ldots, T_{n}(x, y)\right)=\mathcal{T}_{M}(x, y)$ and Eq. (5.7) is verified. The dual construction can also be proved, analogously.

Corollary 5.5. $\left(S_{i M}, \mathcal{T}_{i M}\right)$ is a pair of mutual $N_{S}$-dual functions.

Proof. Straightforward from Proposition 5.3 and 5.6.

Proposition 5.7. Let $M$ be the median aggregation. Additionally, let $\phi, \psi: U \rightarrow U$ be functions in Aut $(U)$ given by $\phi_{r}(x)=x^{r}$ and $\psi_{r}(x)=1-(1-x)^{r}$, respectively. Then, for all $x, y \in U$, the following holds:

$$
\left(\mathcal{T}_{M}\right)^{\phi_{r}}(x, y)=\left(\mathcal{T}_{\phi_{r}(i)}\right)_{M^{\phi_{r}}}(x, y) \quad \text { and } \quad\left(S_{i M}\right)^{\psi_{r}}(x, y)=\left(S_{\psi_{r}(i)^{-1}}\right)_{M^{\psi_{r}}}(x, y) .
$$

Proof. For all $x, y \in U$, based on results from Proposition 5.2 to 3.6, we have that

$$
\begin{aligned}
\left(\mathcal{T}_{M}\right)^{\phi}(x, y) & =\phi^{-1}\left(\mathcal{T}_{M}(\phi(x), \phi(y))\right) \text { by Eq. }(2.2) \\
& =\phi^{-1}\left(M\left(T_{1}(\phi(x), \phi(y))\right), \ldots, T_{n}(\phi(x), \phi(y))\right) \text { by Eq. (3.2) } \\
& =\phi^{-1}\left(M\left(\phi\left(T_{1}^{\phi}(x, y)\right), \ldots, \phi\left(T_{n}^{\phi}(x, y)\right)\right)\right. \text { by Eq. (2.2) } \\
& =\phi^{-1}\left(M\left(\phi\left(T_{1}(x, y)\right), \ldots, \phi\left(T_{\sqrt[r]{n}}(x, y)\right)\right)\right. \\
& =\left(\mathcal{T}_{\phi(i)^{-1}}\right)_{M^{\phi}}(x, y) \text { by Eqs. (3.7) and (3.2) }
\end{aligned}
$$

Therefore, Eq. (5.8)a is verified. The dual construction can also be proved, analogously. 


\subsection{Aggregating fuzzy (S,N)-subimplications}

This section describes the class of aggregating fuzzy (S,N)-subimplications obtained by considering the median operator.

Proposition 5.8. [24, Proposition 12] Let $\mathcal{I}_{M}$ be the (M, I)-operator defined by the median aggregation $M$ and the family $\mathcal{I}_{S, N}$ of $(S, N)$ )-subimplications, which was previously defined in Eq. (4.2). Then, $\mathcal{I}_{M}$ satisfies $\mathbf{I 0}, \mathbf{I 2}, \mathbf{I 3}, \mathbf{I 4}, \mathbf{I 6}, \mathbf{I 7}$ and $\mathbf{I 8}$ when all the member functions of $I_{i} \in \mathcal{I}$ satisfies I0, I2, I3, I4, I6, I7andI8, respectively.

Proposition 5.9. [24, Proposition 13] The (M, I)-operator defined by the median aggregation $M$ and the family of $(S, N)$ )-subimplications $\mathcal{I}$, which was previously defined in Eq. (4.2), satisfies I5.

Proposition 5.10. [24, Corollary 3] Let (A, I)-operator be the median aggregation $M$ and $\mathcal{I}$ $(\mathcal{J})$ be the family of (S,N)-sub(co)implications previously defined by Eq.(4.2). The operators $\mathcal{I}_{M}$ and $\mathcal{J}_{M}$ given by

$$
\mathcal{I}_{M}(x, y)=S_{M}(N(x), y) \text { and } \mathcal{J}_{M}(x, y)=\mathcal{T}_{M}(N(x), y),
$$

are a $\left(S_{M}, N\right)$-subimplication and a $\left(\mathcal{T}_{M}, N\right)$-subcoimplication, respectively.

Proposition 5.11. For all $x, y \in U$, the following functions

$$
\begin{aligned}
& \left(S_{i M, N_{S}}\right)(x, y)=\left(\mathcal{I}_{S_{i}, N_{S}}\right)_{M}(x, y) \text { and } \\
& \left(\mathcal{T}_{i M, N_{S}}\right)(x, y)=\left(\mathcal{J}_{\mathcal{T}_{i}, N_{S}}\right)_{M}(x, y),
\end{aligned}
$$

are $a\left(S_{i M}, N_{S}\right)$-subimplication and a $\left(\mathcal{T}_{i M}, N_{S}\right)$-subcoimplication, respectively.

Proposition 5.13 summarizes the main results related to an $\mathcal{I}_{M}\left(\mathcal{J}_{M}\right)$ S-sub(co)implication, and the diagram presented in Figure 3 shows that the median aggregation $M$ preserves the $(\mathrm{S}, \mathrm{N})$ subimplication class defined in Proposition 5.8, which means, $\mathcal{I}_{S_{M}, N_{S}}$ is also an (S,N)-subimplication. Analogously, we can obtain it by aggregating subimplications in the S-subimplication class.

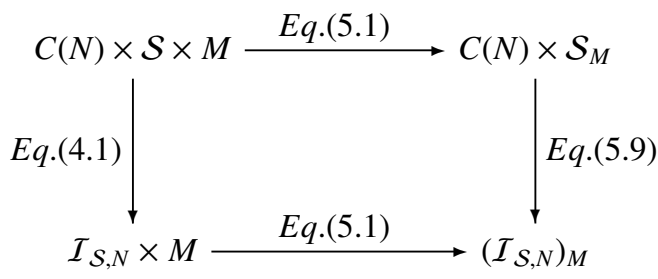

Figure 3: $\left(S_{M}, N_{S}\right)$-implication class. 


\subsubsection{Conjugate and dual (S,N)-sub(co)implications obtained by the median}

Let the $(A, \mathcal{I})$-operator be the median aggregation $M$ and $\mathcal{J}$ be the family of $(\mathrm{S}, \mathrm{N})$-subcoimplications obtained by the dual construction. The operator $\mathcal{I}_{M}$ is a $\left(\mathcal{T}_{M}, N_{S}\right)$-subcoimplication whose expression is $\mathcal{J}_{M}(x, y)=\mathcal{T}_{M}\left(N_{S}(x), y\right)$.

In the following, we consider an automorphism $\phi: U \rightarrow U$ together with the subclass $S_{P_{i}}$ of t-subconorms obtained by the median aggregation in order to present conjugate functions which are preserved by $(\mathrm{S}, \mathrm{N})$-subimplications also obtained by the median aggregation. The corresponding dual construction is also discussed.

Proposition 5.12. Let $\phi_{r}, \psi_{r}: U \rightarrow U$ be functions in Aut $(U)$ given by $\phi_{r}(x)=x^{r}$ and $\psi_{r}(x)=1-(1-x)^{r}$, respectively. Then, for all $x, y \in U$, the following holds:

$$
\begin{aligned}
& \left(\mathcal{I}_{S_{i}, N_{S} M}\right)^{\psi_{r}}(x, y)=\left(\mathcal{I}_{S_{i} \psi_{r}, N_{S}^{\psi_{r}}}\right)_{M^{\psi_{r}}}(x, y) \quad \text { and } \\
& \left(\mathcal{J}_{\mathcal{T}_{i}, N_{S} M}\right)^{\phi_{r}}(x, y)=\left(\mathcal{J}_{\mathcal{T}_{i} \phi_{r}, N_{S}^{\psi_{r}}}\right)_{M^{\phi_{r}}}(x, y) .
\end{aligned}
$$

Proof. For all $x, y \in U$, by Propositions 4.13 and 5.6 and Eq. (5.7), we have that

$$
\begin{aligned}
\left(\mathcal{I}_{S_{i}, N_{S} M}\right)^{\psi_{r}}(x, y) & =\left(S_{i, N_{S}^{\psi_{r}} M}\right)^{\psi_{r}}(x, y) \\
& =S_{P_{M}^{\psi_{r}}}^{\psi_{r}}\left(N_{S}^{\psi_{r}}(x), T_{P}^{\psi_{r}}(x, y)\right) \\
& =\left(\mathcal{I}_{S_{i} \psi_{r}, N_{S}^{\psi_{r}}}\right)_{M^{\psi_{r}}}(x, y) .
\end{aligned}
$$

Therefore, Eq. (5.7) is verified. Its dual construction can also be proven, analogously.

\subsection{Aggregating fuzzy QL-subimplications}

This section analyzes under which conditions the class of fuzzy QL-subimplications are preserved by the median aggregation operator, investigating properties. We present the subclass of fuzzy QL-subimplication represented by a t-norm $T_{P}$, the standard negation $N_{S}$ together with a t-subconorm $S_{P}$, obtained by aggregating t-subconorms of the family $S_{P}$.

Proposition 5.13. [24, Proposition 14] Let $N$ be a fuzzy negation and $M: U^{n} \rightarrow U$ be the median aggregation operator. Then $\mathcal{I}_{M}\left(\mathcal{J}_{M}\right): U^{2} \rightarrow U$ given by

$$
\mathcal{I}_{M}(x, y)=\mathcal{I}_{S_{M}, T, N}(x, y) \quad \text { and } \quad \mathcal{J}_{M}(x, y)=\mathcal{J}_{\mathcal{T}_{M}, S, N}(x, y),
$$

is a $Q L$-sub(co)implicator in $\mathcal{I}_{Q L}\left(\mathcal{J}_{Q L}\right)$.

Analogously, the following results can be stated for an $(\mathcal{J}, M)$-operator obtained by the median aggregation operator acting over a set of fuzzy QL-subcoimplicators.

Corollary 5.6. Let $M: U^{n} \rightarrow U$ be the median aggregation and $\left\{J_{i}: U^{k} \rightarrow U\right\}$ be a family of QL-subimplications given by Eq. (4.4). Then $\mathcal{I}_{M}$ satisfies I0, I3, I4, I7 and $\mathbf{I 8 .}$ 
Proof. Straightforward from Propositions 4.9 and 5.13.

Corollary 5.7. For all $x, y \in U$ and the following holds:

$$
\begin{aligned}
& \mathcal{I}_{S_{i M}, T_{P}, N_{S}}(x, y)=\left(\mathcal{I}_{S_{i}, T_{P}, N_{S}}\right)_{M}(x, y) \text { and } \\
& \mathcal{J}_{\mathcal{T}_{i_{M}}, S_{P}, N_{S}}(x, y)=\left(\mathcal{J}_{\mathcal{T}_{i}, S_{P}, N_{S}}\right)_{M}(x, y) .
\end{aligned}
$$

Additionally, $I_{S_{i}, T_{P}, N_{S}} \in \mathcal{I}_{Q L}$ and $J_{\mathcal{T}_{i M}, S_{P}, N_{S}} \in \mathcal{J}_{Q L}$, respectively.

Proof. Straightforward.

In Figure 4, a diagrammatic representation of the result stated in Proposition 5.13 is presented. In this graphical description we see that the median aggregation $M$ preserves the fuzzy QLsubimplication class, meaning that the following statements are equivalent:

(i) First, by applying the median operator to the family $S_{P}$ we get $S$. Thus, we are able to define an $(\mathcal{J}, A)$-operator as a fuzzy QL-subimplication represented by a t-norm $T_{P}$ and the standard negation $N_{S}$ together with a t-subconorm $S$.

(ii) For each t-subconorm $S_{i}$, the family $\mathcal{J}$ of QL-implications whose explicitly representable member-functions are given by $I_{S_{i}, T_{P}, N_{S}}$, are constructed. Thus, as a consequence, by aggregating $n$ member-functions of $\mathcal{J}$, we obtain an $(\mathcal{J}, A)$-operator.

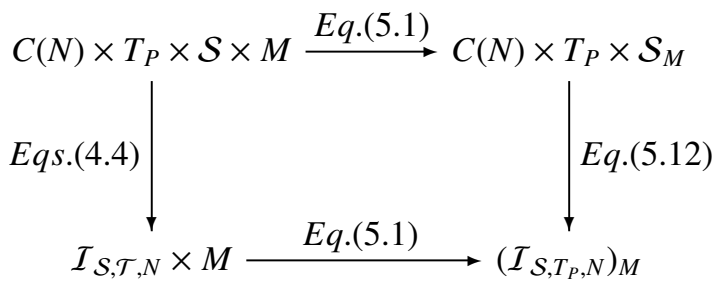

Figure 4: $\left(S_{\mathcal{A}}, \mathcal{T}_{\mathcal{A}}, N\right)$-implication class obtained by the median aggregation operator.

\subsubsection{Conjugate and dual QL-subimplications obtained by the median}

In this section, conjugate and dual QL-sub(co)implications are analysed.

Proposition 5.14. Let $\phi_{r}, \psi_{r}: U \rightarrow U$ be functions in Aut $(U)$ given as $\phi_{r}(x)=x^{r}$ and $\psi_{r}(x)=1-(1-x)^{r}$, respectively. Then, for all $x, y \in U$, the following holds:

$$
\begin{aligned}
& \left(\mathcal{I}_{S_{i}, T_{P}, N_{S} M}\right)^{\psi_{r}}(x, y)=\left(\mathcal{I}_{S_{i} \psi_{r}, T_{P}^{\psi_{r}}, N_{S}^{\psi_{r}}}\right)_{M^{\psi_{r}}}(x, y) \quad \text { and } \\
& \left(\mathcal{J}_{\mathcal{T}_{i}, S_{P}, N_{S} M}\right)^{\phi_{r}}(x, y)=\left(\mathcal{J}_{\mathcal{T}_{i} \phi_{r}, T_{P}^{\phi_{r}}, N_{S}^{\phi_{r}}}\right)_{M^{\phi_{r}}}(x, y) .
\end{aligned}
$$


Proof. For all $x, y \in U$, based on results in Propositions 4.13, 5.7 and 5.13, we have that

$$
\begin{aligned}
\left(\mathcal{I}_{S_{i}, T_{P}, N_{S} M}\right)^{\psi_{r}}(x, y) & =\left(S_{P_{i}, T_{P}, N_{S} M}\right)^{\psi_{r}}(x, y) \\
& =S_{P_{i}^{\psi_{r}} M^{\psi_{r}}}\left(N_{S}^{\psi_{r}}(x), T_{P}^{\psi_{r}}(x, y)\right) \\
& =\left(\mathcal{I}_{S_{P_{i}}{ }^{\psi_{r}}, T_{P}^{\psi_{r}}, N_{S}^{\psi_{r}}}\right)_{M^{\psi_{r}}}(x, y) .
\end{aligned}
$$

So, the aggregator $M$ preserves the $\psi_{r}$-conjugate of an QL-subimplication $\mathcal{I}_{S_{i}, T_{P}, N_{S} M}$. Its dual construction can also be proved, in an analogous manner.

Proposition 5.15. For all $x, y \in U$, the following holds:

$$
\left(\mathcal{I}_{S_{i}, T_{P}, N_{S}}\right)_{N_{S}}(x, y)=\mathcal{J}_{S_{i}, T_{P}, N_{S}}(x, y) \quad \text { and } \quad\left(\mathcal{J}_{\mathcal{T}_{i}, S_{P}, N_{S}}\right)_{N_{S}}(x, y)=\mathcal{I}_{\mathcal{T}_{i}, S_{P}, N_{S}}(x, y) .
$$

\section{CONCLUSION AND FINAL REMARKS}

In this paper we characterize both $(\mathrm{S}, \mathrm{N})$ - and QL-subimplications with respect to the median aggregation operator. In particular, the underlying principle of the proof related to properties preserved by the new (S,N)- and QL-subimplications obtained by the median aggregation is obtained in a similar methodology to the $(\mathrm{S}, \mathrm{N})$ - and QL-implications. Since such classes of subimplication are represented by t-subconorms and t-subnorms which are characterized by generalized associativity, the corresponding (S,N)- and QL-subimplications are related by distributive $n$-ary aggregation together with generalizations, as the exchange and neutrality principles. Ongoing work on application of Atanassov's intuitionistic extension of fuzzy connectives provides relevant methods to obtain other operators by distributive $n$-ary aggregation.

\section{ACKNOWLEDGMENTS}

This work was supported by the Brazilian funding agencies CAPES, CNPq and FAPERGS (Eds. 309533/2013-9 (PQ-2 CNPq), 309533/2013-9 (PqG FAPERGS) e 448766/2014-0 (MCTI/ CNPQ/Universal 2014/B).

RESUMO. Neste trabalho, (S,N)- e QL-subimplicações são obtidas por aplicação de operadores $n$-arios de agregação sobre as classes $\mathcal{T}$ de t-subnormas e $S$ de t-subconormas, considerando negações fuzzy involutivas. As classes de (S,N)- e QL-subimplicações são assim explicitamente representadas por t-subconormas e t-subnormas que verificam a associatividade generalizada. As correspondentes classes de subimplicações $I_{S, N}$ e $I_{S, T, N}$, são caracterizadas por agregações distributivas que satisfazem o princípio da troca e da neutralidade. Neste contexto, analisam-se as classes de (S,N)- e QL-subimplicações, as quais são obtidas pela ação do operador mediana, considerando a negação padrão $N_{S}$ e a família de tsubnormas e t-subconormas, respectivamente geradas pelo produto $T_{P}$ e soma algébrica $S_{P}$. Como principal resultado, mostra-se que as família $I_{S_{P}, N}$ e $I_{S_{P}, T_{P}, N}$ preservam propriedades, estendendo os principais relacionamentos das correspondentes classes de ( $\mathrm{S}, \mathrm{N})$ - e QL-implicações fuzzy, discutindo ainda as construções duais e as formas conjugadas obtidas por ação de automorfismos. 
Palavras-chave: Mediana, agregações, t-sub(co)normas, fuzzy (sub)implicações, QL-implicações, (S,N)-implicações.

\section{REFERENCES}

[1] G. Mayor \& J. Monreal. Additive generators of discrete conjunctive aggregation operations. IEEE Transactions on Fuzzy Systems, 15(6) (2007), 1046-1052.

[2] S. Rasheed, D. Stashuk \& M.S. Kamel. Integrating heterogeneous classifier ensembles for EMGsignal decomposition based on classifier agreement. IEEE Transactions on Fuzzy Systems, 14(3) (2010), 866-882.

[3] H. Izakian, W. Pedrycz \& I. Jam. Clustering spatio temporal data: an augmented fuzzy C-Means. IEEE Transaction on Fuzzy Systems, 21(5) (2013), 855-868.

[4] B. Geng, J.K. Mills \& D. Sun. Two-Stage charging strategy for plug-In electric vehicles at the residential transformer level. IEEE Transaction on Smart Grid, 4(3) (2013), 1442-1452.

[5] E.P. Klement, R. Mesiar \& E. Pap. Triangular Norms, Dordrecht: Kluwer Academic Publisher, (2000).

[6] T. Calvo, A. Kolesárová, M. Kormoníková \& R. Mesiar. Aggregation operators: Properties, classes and construction methods, in Aggregation Operators New Trends and Applications (T. Calvo, G. Mayor \& R. Mesiar, Eds.), Studies in Fuzziness and Soft Computing, Springer, 97 (2002), 3-106.

[7] V. Torra. Aggregation operators and models. Fuzzy Sets and Systems, 156(3) (2005), 407-410.

[8] H. Bustince, T. Calvo, B.D. Baets, J.C. Fodor, R. Mesiar, J. Monteiro, D. Paternain \& A. Pradera. A class of aggregation fucntions encopassing two-dimensional OWA operations. Information Sciences, 180(10) (2010), 1977-1989.

[9] G. Beliakov, H. Bustince \& J. Fernandez. The median and its extensions. Fuzzy Sets and Systems, 1175 (2011), 36-47.

[10] J. Wang, K. Li \& H. Zhang. Multi-criteria decision-making method based on induced intuitionistic normal fuzzy related aggregation operators. Intl. Journal of Uncertainty, Fuzziness and KnowledgeBased Systems, 20(4) (2012), 559-578.

[11] G. Cornelis, G. Deschrijver \& E. Kerre. Implications in intuitionistic fuzzy and interval-valued fuzzy set theory: construction, classification and application. Intl. Journal of Approximate Reasoning, 35 (2004), 55-95.

[12] B. Bedregal, G. Dimuro, R. Santiago \& R. Reiser. On interval fuzzy S-implications. Information Sciences, 180 (2010), 1373-1389.

[13] G. Dimuro, B. Bedregal, R. Santiago \& R. Reiser. Interval additive generators of interval t-norms and interval t-conorms. Inf. Sciences, 181 (2011), 3898-3916.

[14] G. Beliakov, H. Bustince, S. James, T. Calvo \& J. Fernandez. Aggregation for Atanassov's intuitionistic and interval valued fuzzy sets: the median operator. IEEE Transactions on Fuzzy Systems, 20 (2012), 487-498.

[15] R. Reiser \& B. Bedregal. Interval-valued intuitionistic fuzzy implications - construction, properties and representability. Information Sciences, 248 (2013), 68-88.

[16] D. Li, L. Wang \& G. Chen. Group decision making methodology based on the Atanassov's intuitionistic fuzzy set generalized OWA operator. Intl. Journal of Uncertainty, Fuzziness and KnowledgeBased Systems, 18(6) (2010), 801-817. 
[17] J. Lin \& Q. Zhang. Some continuous aggregation operators with interval-valued intuitionistic fuzzy Information and their application to decision making. Intl. Journal of Uncertainty, Fuzziness and Knowledge-Based Systems, 20(2) (2012), 185-209.

[18] H. Bustince, E. Barrenechea \& M. Pagola. Generation of interval-valued fuzzy and Atanassov's intuitionistic fuzzy connectives from fuzzy connectives and from $k_{\alpha}$ operators: Law of conjuntions and disjuntions, amplitute. International Journal of Intelligent Systems, 23 (2008), 680-714.

[19] M. Xia, Z. Xu \& N. Chen. Some hesitant fuzzy aggregation operators with their application in group decision making. Group Decision and Negotiation, 22(2) (2013), 259-279.

[20] B. Bedregal, R. Reiser, H. Bustince, C. Lopez-Molina \& V. Torra. Aggregation functions for typical hesitant fuzzy elements and the action of automorphisms. Information Sciences, 255(1) (2014), 82-99.

[21] M. Grabisch, J. Marichal, R. Mesiar \& E. Pap. Aggregation functions: Means. Information Sciences, 181 (2011), 1-22.

[22] R. Reiser, B. Bedregal \& M. Baczyński. Aggregating fuzzy implications. Information Sciences, 253 (2013), 126-146.

[23] A. Mesiarová. Continuous triangular subnorms. Fuzzy Sets and Systems, 42 (2004), 5-83.

[24] I. Benitez, R. Reiser, A. Yamin \& B. Bedregal. Aggregating Fuzzy QL-Implications. IEEE Xplore Digital Library Pos-Proc. of 3rd Workshop-School on Theoretical Computer Science, pp. 121-128, DOI: 10.1109/WEIT.2013.11.

[25] Y. Shi, B.V. Gasse, D. Ruan \& E.E. Kerre. On the first place antitonicity in QL-implications. Fuzzy Sets and Systems, 159(22) (2008), 2988-3013.

[26] E. Klement, R. Mesiar \& E. Pap. Triangular norms. Position paper I: basic analytical and algebraic properties. Fuzzy Sets and Systems, 143(1) (2004), 5-26.

[27] M. Baczyński \& B. Jayaram. QL-implications: Some properties and intersections. Fuzzy Sets and Systems, 161 (2010), 158-188.

[28] L. Kitainik. Fuzzy Decision Procedures with Binary Relations, Dordrecht: Kluwer Academic Publisher (1993).

[29] H. Bustince, P. Burillo \& F. Soria. Automorphisms, negations and implication operators. Fuzzy Sets and Systems, 134(2) (2003), 209-229.

[30] M. Baczyński \& B. Jayaram. (S,N)- and R-implications: A state-of-the-art survey. Fuzzy Sets and Systems, 159(14) (2008), 1836-1859.

[31] M. Mas, M. Monserrat \& J. Torrens. On interval fuzzy negations. Fuzzy Sets and Systems, 158 (2007), 2612-2626.

[32] G. Maksa. Quasisums and generalized associativity. Aequationes Mathematicae, 69(1-2) (2005), 6-27.

[33] J. Fodor \& I.J. Rudas. An extension of the migrative property for triangular norms. Fuzzy Sets and Systems, 168(1) (2011), 70-80.

[34] B. Bedregal. A normal form which preserves tautologies and contradictions in a class of fuzzy logics. J. Algorithms, 62 (2007), 135-147.

[35] M. Mas, M. Monserrat \& J. Torrens. QL-implications versus D-implications. Kybernetika, 42(3) (2006), 351-366. 